\title{
Direct quantification of lipopeptide biosurfactants in biological samples via HPLC and UPLC-MS requires sample modification with an organic solvent
}

\author{
Piotr Biniarz ${ }^{1}$ - Marcin Lukaszewicz ${ }^{1}$ \\ Received: 30 December 2016 /Revised: 9 March 2017 / Accepted: 20 March 2017 / Published online: 22 April 2017 \\ (C) The Author(s) 2017. This article is an open access publication
}

\begin{abstract}
The rapid and accurate quantification of biosurfactants in biological samples is challenging. In contrast to the orcinol method for rhamnolipids, no simple biochemical method is available for the rapid quantification of lipopeptides. Various liquid chromatography (LC) methods are promising tools for relatively fast and exact quantification of lipopeptides. Here, we report strategies for the quantification of the lipopeptides pseudofactin and surfactin in bacterial cultures using different high- (HPLC) and ultra-performance liquid chromatography (UPLC) systems. We tested three strategies for sample pretreatment prior to LC analysis. In direct analysis (DA), bacterial cultures were injected directly and analyzed via LC. As a modification, we diluted the samples with methanol and detected an increase in lipopeptide recovery in the presence of methanol. Therefore, we suggest this simple modification as a tool for increasing the accuracy of LC methods. We also tested freeze-drying followed by solvent extraction (FDSE) as an alternative for the analysis of "heavy" samples. In FDSE, the bacterial cultures were freeze-dried, and the resulting powder was extracted with different solvents. Then, the organic extracts were analyzed via LC. Here, we determined the influence of the extracting solvent on lipopeptide recovery. HPLC methods allowed us to quantify pseudofactin and surfactin with run times of 15 and 20 min per sample, respectively, whereas UPLC quantification was as fast as 4 and 5.5 min per sample, respectively. Our methods provide highly accurate measurements and high recovery levels for lipopeptides. At the same time, UPLC-MS provides the
\end{abstract}

Marcin Łukaszewicz

marcin.lukaszewicz@uwr.edu.pl

1 Department of Biotransformation, Faculty of Biotechnology, University of Wroclaw, Joliot Curie 14a, 50-383 Wroclaw, Poland possibility to identify lipopeptides and their structural isoforms.

Keywords Biosurfactants · Lipopeptides · Quantification · HPLC · UPLC-MS · Extraction

\section{Introduction}

Biosurfactants (BS) are surface-active compounds of microbial origin. BS molecules consist of a hydrophobic "tail," which is usually a fatty acid or $\beta$-hydroxyl fatty acid of 4-18 carbon atoms, and hydrophilic "head." A wide range of molecules are considered to be BS, and classification is made primarily based on the chemical nature of the hydrophilic part of the molecule. Therefore, BS are divided into several groups: lipopeptides (LPs), glycolipids, lipoproteins, phospholipids, and polysaccharides. Over the years, BS have gained the attention of researchers around the world. BS are considered to be environmentally friendly substitutes for synthetic surfactants. Moreover, BS can act as bioemulsifiers, antibiotics, antifungals, heavy metal-binding compounds, or antitumor compounds. Therefore, BS can be utilized in different fields, such as industry, environmental protection, medicine, or farming. Potential applications of BS and LPs have been the subject of a number of reviews and research articles (Das et al. 2010; Banat et al. 2010; Janek et al. 2010, 2012; Gudiña et al. 2013; Janek et al. 2013; Duarte et al. 2014).

LPs appear to be a particularly promising class of BS because they can exhibit a variety of possible structures. The hydrophilic peptide moiety of known LPs consists of 4 to 25 amino acids, which can also form a lactone ring. The modularity of the lipopeptides' structures results in a broad spectrum of properties and activities (Mulligan 2005; Mukherjee 
and Das 2010; Banat et al. 2010; Soberon-Chavez and MillerMaier 2011; Jurado et al. 2012; Biniarz et al. 2016).

To date, LPs synthesized by different strains of Bacillus or Pseudomonas have been studied extensively, and several LP families have been mentioned in the literature: surfactins, iturins, fengycins, lychenisins, viscosins, amphisins, and several others (Raaijmakers et al. 2006; Das et al. 2010; Banat et al. 2010; Mnif and Ghribi 2015). Surfactin (SU), which is produced by a number of Bacillus subtilis isolates, is the best known and most extensively studied LP. SU is produced as a mixture of structural analogs that differ in the length and branching of the carbon chain, as well as in substitutions in the amino acids of the hydrophilic head. In addition, the abundance ratio of the analogs can differ among $B$. subtilis strains and change in response to culture conditions (Akpa et al. 2001; De Faria et al. 2011; Ben Ayed et al. 2014; Jajor et al. 2015; Mnif and Ghribi 2015). The physiochemical and biological properties of SU have been investigated extensively by many authors, revealing the antibacterial, antifungal, anticancer, heavy metal-binding, and emulsifying activities of SU (Mukherjee and Das 2010; Banat et al. 2010; Gudiña et al. 2013; Duarte et al. 2014). Research on the properties of SU is typically carried out using a mixture of SU analogs, as the separation of individual analogs can be challenging (Kowall et al. 1998; Banat et al. 2010; Tang et al. 2010). Pseudofactin (PF) is a cyclic LP that is produced by the Arctic Pseudomonas fluorescens strain BD5 (Janek et al. 2010). The PF molecule consists of a saturated linear fatty acid linked to a peptide head of eight amino acids. Initially, two PF analogs were characterized. PF1 $\left(\mathrm{C}_{16}-\mathrm{Val}_{8}\right)$ and PF2 $\left(\mathrm{C}_{16}-\mathrm{Leu}_{8}\right)$ differ in only one amino acid in the eighth position (Janek et al. 2010). P. fluorescens BD5 is also able to produce two other PF analogs, which were identified later: PF3 $\left(\mathrm{C}_{18}-\mathrm{Val}_{8}\right)$ and PF4 $\left(\mathrm{C}_{18}-\mathrm{Leu}_{8}\right)$ (Biniarz et al. 2015b). Of these four known analogs, PF2 is the most abundant when $P$. fluorescens BD5 is cultivated on minimal medium (Janek et al. 2010), but the ratio between the analogs changes in response to culture conditions (Biniarz et al. 2015b). The physiochemical and biological properties of PF2 were investigated. PF2 exhibits good emulsification activity in comparison to synthetic detergents (Janek et al. 2010), as well as antimicrobial, antiadhesive, and antibiofilm activity against several uropathogenic bacterial strains and Candida albicans (Janek et al. 2012; Biniarz et al. 2015a; Janek et al. 2016). Moreover, PF2 exhibits strong antitumor activity (Janek et al. 2013). There is a great need to investigate the properties of other $\mathrm{PF}$ analogs; therefore, methods for the exact identification and quantification of LP analogs should be established.

In recent years, extensive efforts have been made to isolate and characterize BS, as well as to investigate possible industrial applications for these molecules. However, the rapid and reliable quantification of BS remains challenging. Studies of the properties of $\mathrm{BS}$, as well as the optimization of the production and utilization of BS in industry, cosmetics, drugs, etc., require fast and accurate tools for their quantification. The exact determination of the ratios between BS analogs is also of great importance.

BS have long been quantified indirectly. Several methods based on measuring changes in the surface properties of BS water solutions have been validated and utilized. These methods include surface tension measurements (Youssef et al. 2004; Joshi et al. 2013), drop-collapse assays (Youssef et al. 2004; Chen et al. 2007; Burch et al. 2010), critical micelle dilution (CMD) (Youssef et al. 2004; Satpute et al. 2008), the microplate meniscus shape assay (Chen et al. 2007), and turbidometric methods (Mukherjee et al. 2009). However, these methods can only be used as semi-quantitative techniques at best (Marchant and Banat 2014; Rudden et al. 2015; Biniarz et al. 2016). Simple colorimetric methods were also developed for the quantification of specific groups of BS and LPs. Perhaps the best known colorimetric method is the orcinol method for measuring rhamnose content in samples containing rhamnolipids. However, in this case, the results often appear to be overestimated (Marchant and Banat 2014; Rudden et al. 2015). To our knowledge, there is no colorimetric test that is specific for all LPs. Anionic BS and LPs can be detected via the blue agar plate method, but to our knowledge, this method has been utilized only as a qualitative approach (Satpute et al. 2010). Recently, a polydiacetylene (PDA) vesicle colorimetric test for the quantification of SU was developed (Zhu et al. 2014). The authors stated that PDA vesicles are a high-throughput and accurate method for the quantification of ionic surfactants (Zhu et al. 2014), but this method has been cited in a limited number of research papers to date and has not been tested for other BS.

Liquid chromatography (LC) is a powerful tool for the identification and quantification of active compounds in biological samples. Reports on the quantification of BS via reverse-phase high-performance liquid chromatography (RPHPLC) and reverse-phase ultra-performance liquid chromatography (RP-UPLC) appeared recently (Rudden et al. 2015). LC provides researchers with highly sensitive and accurate measurements. In addition, LC enables the characterization of BS mixture components (e.g., the relative abundance of individual analogs in a mixture). LC systems can be also coupled with mass spectrometry (MS) or tandem MS (MS/ MS). This modification enables the reliable identification and structural characterization of BS (Marchant and Banat 2014; Rudden et al. 2015).

Although some researchers inject LP samples directly onto HPLC/UPLC columns (Davis et al. 2001), the quantification of LPs by LC is more often preceded by laborious sample pretreatment (Rao et al. 2008). Acid precipitation (Hsieh et al. 2008; Yokota et al. 2012) or different solvent mixtures (Romero et al. 2007; Yuan et al. 2011; Yokota et al. 2012) are commonly used to extract LPs from culture supernatants. 
Next, the extracts are dried, dissolved in an organic solvent (usually methanol), and analyzed via LC. An organic solvent matrix provides good solubility for LPs, but this approach has several drawbacks: (1) the recovery levels of LPs from culture supernatants tend to be undefined and (2) sample pretreatment for high-throughput LC analysis should be minimized. Therefore, it is of great importance to investigate and validate quantitative methods for LPs that would minimize the sample pretreatment process. On the other hand, the direct injection of non-pretreated biological samples onto LC columns can result in adsorption issues for peptides and LPs and poor recovery levels of active substances, as well as damage to RP-HPLC columns (van den Broek et al. 2008; Yokota et al. 2012).

The aim of this study was to develop and validate fast, reliable, and simple methods for the quantification of LPs in bacterial cultures with different HPLC and UPLC-MS systems and columns, using PF and SU as standards. Three different protocols for sample pretreatment were tested. In direct analysis (DA), bacterial cultures were clarified by centrifugation and then directly injected and analyzed by LC. As a modification of DA, the clarified samples were diluted with methanol $(\mathrm{MeOH})$. We showed that the recovery levels of LPs during HPLC and UPLC-MS analysis depend on the concentration of LPs in aqueous samples and the solvent mixture used to dissolve or dilute the sample. As an alternative to DA, freeze-drying followed by solvent extraction (FDSE) was tested for the analysis of "heavy" samples, as a high protein concentration can be damaging to RP-HPLC/UPLC columns (van den Broek et al. 2008). In FDSE, clarified bacterial cultures were freeze-dried, and the resulting powder was extracted with different solvents. Then, the organic extracts were analyzed via LC. The influence of solvent extraction on LP recovery was evaluated.

To our knowledge, this work is the first time that DA and FDSE have been developed and validated for the quantification of LPs via HPLC and UPLC-MS. Moreover, the influence of the LP concentration and $\mathrm{MeOH}$ addition to the sample on the recovery of LPs have been evaluated, highlighting the need for sample dilution with $\mathrm{MeOH}$ prior to LC analysis.

\section{Materials and methods}

\section{Strains and culture conditions}

P. fluorescens BD5 (PCM B/00115) (Janek et al. 2010) and B. subtilis Natto KB1 (PCM B/00114) isolates (both from the Laboratory of Biotransformation, University of Wroclaw culture collection) were grown on Luria-Bertani agar plates (LB; $10 \mathrm{~g} / \mathrm{L}$ of tryptone, $5 \mathrm{~g} / \mathrm{L}$ of yeast extract, and $10 \mathrm{~g} / \mathrm{L}$ of $\mathrm{NaCl}$ ) at $30{ }^{\circ} \mathrm{C}$. Next, single colonies from the agar plates were used to inoculate $10 \mathrm{ml}$ of liquid precultures in LB medium. The precultures were incubated overnight at $30{ }^{\circ} \mathrm{C}$ with agitation
(180 rpm). After growth, the bacteria were pelleted (15 min., $10,000 \times g$ ), washed twice with $0.9 \% \mathrm{NaCl}$, and resuspended in $5 \mathrm{ml}$ of $0.9 \% \mathrm{NaCl}$. The optical density (OD) at $600 \mathrm{~nm}$ was measured with a Hach Oddyssey DR/2500 spectrophotometer, and suspensions were used to inoculate cultures for LP production.

\section{Production of lipopeptides}

The PF-producing strain P. fluorescens BD5 was cultivated in King's B medium: $20 \mathrm{~g} / \mathrm{L}$ of proteose peptone (Becton Dickinson, USA), $1.5 \mathrm{~g} / \mathrm{L}$ of $\mathrm{K}_{2} \mathrm{HPO}_{4}$ (POCH, Poland), $1.5 \mathrm{~g} / \mathrm{L}$ of $\mathrm{MgSO}_{4} \times 7 \mathrm{H}_{2} \mathrm{O}(\mathrm{POCH}$, Poland), and $100 \mathrm{mM}$ MOPS (Bioshop, Canada), supplemented with $4 \%(w / v)$ glycerol (VWR International, USA). The SU-producing strain $B$. subtilis $\mathrm{KB} 1$ was cultivated in modified Landy's medium: $20 \mathrm{~g} / \mathrm{L}$ of glucose (POCH, Poland), $2.3 \mathrm{~g} / \mathrm{L}$ of $\left(\mathrm{NH}_{4}\right)_{2} \mathrm{SO}_{4}$ (POCH, Poland), $2 \mathrm{~g} / \mathrm{L}$ of glutamic acid (POCH, Poland), $1 \mathrm{~g} / \mathrm{L}$ of yeast extract (Becton Dickinson, USA), $0.5 \mathrm{~g} / \mathrm{L}$ of $\mathrm{MgSO}_{4}$ (POCH, Poland), $0.5 \mathrm{~g} / \mathrm{L}$ of $\mathrm{KCl}$ (POCH, Poland), $1.6 \mathrm{mg} / \mathrm{L}$ of $\mathrm{CuSO}_{4}\left(\mathrm{POCH}\right.$, Poland), $1.2 \mathrm{mg} / \mathrm{L}$ of $\mathrm{Fe}_{2}\left(\mathrm{SO}_{4}\right)_{3}$ (POCH, Poland), $0.4 \mathrm{mg} / \mathrm{L}$ of $\mathrm{MnSO}_{4}$ (POCH, Poland), and $100 \mathrm{mM}$ (Bioshop, Canada) (Guez et al. 2008). The cultures were inoculated to achieve an OD of 0.1 and incubated for 3 days at $30^{\circ} \mathrm{C}$ with agitation (180 rpm).

Three different Erlenmeyer flasks and filling volumes were used to culture the bacteria to achieve various oxygenation levels, resulting in different concentrations of LPs at the end of the incubation period: $400 \mathrm{ml}$ of medium in a $1 \mathrm{~L}$ flask (culture A), $200 \mathrm{ml}$ of medium in a $1 \mathrm{~L}$ flask (culture B), and $100 \mathrm{ml}$ of medium in a $0.5 \mathrm{~L}$ baffled flask (culture $\mathrm{C}$ ).

At the end of the incubation period, the cultures were centrifuged (15 min, $10,000 \times g$ ), and the supernatants were used for the designated experiments.

\section{Preparation of lipopeptide standards}

PF was produced and purified as described previously (Janek et al. 2010), with modifications. Briefly, $500 \mathrm{ml}$ of BD5 cellfree culture supernatant (culture A) was extracted three times with ethyl acetate. The solvent was evaporated under a vacuum, and the crude extract was dissolved in $\mathrm{MeOH}$ and purified via RP-HPLC. Semi-preparative RP-HPLC consisted of a Beckman Coulter System Gold 126NMP Pump and a Knauer Variable Wavelength Monitor equipped with a Phenomenex Luna C18(2) column $(100 \mathrm{~mm} \times 30 \mathrm{~mm}$, $10 \mu \mathrm{m})$ under the control of the LP-Chrom software (Lipopharm, Poland). Mobile phases of water with $0.1 \%(v /$ v) TFA (A) and acetonitrile (ACN) with $0.1 \%(v / v)$ TFA (B) were used. The absorbance at $210 \mathrm{~nm}$ was monitored during HPLC preparation. Two milliliters of sample were injected onto a column and eluted with a 40-min gradient (\% A:B $v / v)$ : injection start $(30: 70), 5 \min (30: 70), 10 \min (10: 80)$, 
$20 \min (20: 80), 21 \min (0: 100), 31 \min (0: 100), 32 \min$ (30:70), and $40 \mathrm{~min}$ (30:70). The flow rate was set at $10 \mathrm{ml} /$ min. All observed PF fractions were collected together, freezedried, and weighed. $\mathrm{PF}$ was dissolved in $\mathrm{MeOH}$ and used as a standard stock solution $(1 \mathrm{mg} / \mathrm{ml})$. The SU standard was purchased from Sigma-Aldrich (USA), dissolved in $\mathrm{MeOH}$ and used as a standard stock solution $(1 \mathrm{mg} / \mathrm{ml})$.

\section{HPLC and UPLC-MS conditions}

Three different HPLC and UPLC-MS systems were used for the quantification of LPs. System 1 (HPLC) consisted of a Beckman Gold 126 Pump and a Knauer Variable Wavelength Monitor equipped with a Macherey-Nagel C18 Isis column $(50 \mathrm{~mm} \times 4.6 \mathrm{~mm}, 1.8 \mu \mathrm{m})$ under the control of the LP-Chrom software (Lipopharm, Poland). The column was kept at room temperature during the analyses. System 2 (HPLC) consisted of a Waters e2695 pumping module with an autosampler and a 2998 PDA detector, equipped with a Waters C18 Xbridge column $(50 \mathrm{~mm} \times 4.6 \mathrm{~mm}, 2.5 \mu \mathrm{m})$. The column was kept at room temperature during the analyses. System 3 (UPLC-MS) consisted of a Waters Acquity UPLC System with a 2996 PDA detector and a Waters Xevo QTof MS System, equipped with a Waters Acquity BEH C18 column $(100 \mathrm{~mm} \times 2.1 \mathrm{~mm}, 1.7 \mu \mathrm{m})$, which was kept at $40{ }^{\circ} \mathrm{C}$. Mobile phases of water with $0.1 \%(v / v)$ TFA (A) and ACN with $0.1 \%(v / v)$ TFA (B) were used. The absorbance at $210 \mathrm{~nm}$ (system 1) and the absorbance between 200 and $400 \mathrm{~nm}$ (system 2) were monitored during the HPLC analyses. In the case of UPLC-MS analyses, the absorbance between 200 and $400 \mathrm{~nm}$ was monitored simultaneously with the total ion count (TIC). MS analysis was conducted in positive mode ESI. The source temperature was set to $150{ }^{\circ} \mathrm{C}$, and the desolvation gas temperature was $350{ }^{\circ} \mathrm{C}$. Nitrogen was used as the desolvation gas $(800 \mathrm{~L} / \mathrm{h})$ and the cone gas $(20 \mathrm{~L} / \mathrm{h})$. The cone voltage was set to $10 \mathrm{~V}$, and the capillary voltage was set to $3 \mathrm{kV}$. The samples were analyzed in the range of $800-1200 \mathrm{~m} / \mathrm{z}$.

The elution methods used for the quantification of LPs are described below. For system 1, $10 \mu \mathrm{l}$ of sample were injected onto a column and eluted with two different gradients (\% A:B $v / v)$. A 15 min gradient was used for PF: injection start (30:70), $6 \mathrm{~min}(10: 90), 8 \mathrm{~min}(0: 100), 10 \mathrm{~min}(0: 100)$, $12 \mathrm{~min}$ (30:70), and $15 \mathrm{~min}$ (30:70). The flow rate was set to $1 \mathrm{ml} / \mathrm{min}$. For SU, a 20 min gradient was used: injection start (30:70), $2 \min (20: 80), 12 \min (10: 90), 14 \min (0: 100)$, $16 \min (0: 100), 18 \min (30: 70)$, and $20 \min (30: 70)$. The flow rate was set to $1.5 \mathrm{ml} / \mathrm{min}$. For system 2, $50 \mu$ of sample was injected onto a column and eluted with gradients similar to those used for system 1 . For system $3,5 \mu$ of sample was injected onto a column. The elution method for PF was a 4 min gradient (\% A:B) with a flow rate of $0.7 \mathrm{ml} / \mathrm{min}$ : injection start (30:70), $0.5 \mathrm{~min}$ (30:70), $2.5 \mathrm{~min}$ (20:80), $3.0 \mathrm{~min}$
(0:100), $3.5 \min (30: 70)$, and $4.0 \min (30: 70)$. For the analysis of SU, the flow rate was set to $0.6 \mathrm{ml} / \mathrm{min}$ with a $5.5 \mathrm{~min}$ gradient, as follows: injection start (50:50), $0.1 \mathrm{~min}$ (50:50), $1.5 \mathrm{~min}(20: 80), 3.5 \mathrm{~min}(10: 90), 4.5 \mathrm{~min}(0: 100), 5.0 \mathrm{~min}$ (50:50), and $5.5 \min (50: 50)$.

\section{Calibration and validation of HPLC and UPLC-MS methods}

$\mathrm{PF}$ and $\mathrm{SU}$ stock solutions in $\mathrm{MeOH}$ were used to prepare a dilution series of LPs from 0.98 to $1000 \mathrm{mg} / \mathrm{L}$ in $\mathrm{MeOH}$. These samples were injected onto HPLC and UPLC columns and eluted as described above. Each sample was prepared in triplicate and analyzed at least three times. The retention times and peak areas were collected from the HPLC systems for quantification. From the UPLC-MS system, the retention times, peak areas, and TIC at a given $\mathrm{m} / \mathrm{z}$ were collected. The results were used to prepare standard curves for LP quantification and to determine standard deviations (SD), relative standard deviations (RSD), limits of detection (LOD), and limits of quantification (LOQ). For the purpose of PF and SU quantification, all detected $\mathrm{PF}$ or $\mathrm{SU}$ peaks were integrated and summed. The LOD and LOQ were determined based on a visual evaluation of chromatograms, as suggested by the United States Food and Drug Administration (US FDA 1999).

\section{Recovery tests}

Three different methods were tested for the HPLC and UPLCMS quantification of LPs. In DA, LP-containing solutions were clarified by centrifugation $(15 \mathrm{~min}, 10,000 \times \mathrm{g})$ and injected onto HPLC and UPLC-MS columns. Optionally, the samples were diluted with water or $\mathrm{MeOH}$ before injection. For the analysis of FDSE efficiency, $1 \mathrm{ml}$ of supernatants were freeze-dried and then extracted three times with $1 \mathrm{ml}$ of $\mathrm{MeOH}$, ethanol (EtOH), butanol (ButOH), ACN, or ethyl acetate $(\mathrm{EtOAc})$. Next, the solvents were evaporated under a vacuum, and resulting pellet was resuspended in $\mathrm{MeOH}$ and used for further HPLC and UPLC-MS analyses.

Recovery tests for DA were performed as follows: 50, 100, and $250 \mu \mathrm{g}$ of PF or SU from stock solutions were added to Eppendorf test tubes and dried. Then, LB or LB:water (50:50 $v / v)$ or $\mathrm{LB}: \mathrm{MeOH}(50: 50 v / v)$ mixtures were added to a final volume of $1 \mathrm{ml}$. Next, the samples were injected onto HPLC and UPLC-MS columns and analyzed as described above. Similarly, different mixtures of LB:MeOH (90:10, 80:20, $60: 40,40: 60,20: 80$, and 10:90 v/v) were used to dissolve 50 and $250 \mu \mathrm{g}$ of PF or SU from stock solutions to show the effect of the MeOH concentration on LP recovery.

For the analysis of FDSE efficiency, $250 \mu \mathrm{g}$ of PF or SU from stock solutions were added to Eppendorf test tubes and dried. Then, LB was added to a final volume of $1 \mathrm{ml}$. The samples were freeze-dried, and the resulting pellet was 
extracted three times with $1 \mathrm{ml}$ of $\mathrm{MeOH}$, EtOH, ButOH, $\mathrm{ACN}$, or EtOAc by vortex-shaking for $1 \mathrm{~min}$ at room temperature. Next, the solvents were evaporated under a vacuum, and the resulting pellet was resuspended in $\mathrm{MeOH}$ and used for further HPLC and UPLC-MS analyses. A similar protocol was also used for clarified 1-ml samples of culture supernatants after the production of LPs.

\section{Quantification of lipopeptides in bacterial cultures}

As described in the "Production of lipopeptides" section, supernatants were used for the DA and FDSE experiments. When indicated, the samples were diluted with water (50:50 $v / v)$ or $\mathrm{MeOH}(50: 50$ or 10:90 $v / v)$ before HPLC or UPLC-MS quantification. The obtained data (retention times, peak areas, and TIC) were compared to LP standard curves.

\section{Data analysis}

The Microsoft Excel software was used to analyze the obtained data. Means, standard deviations (SD), and relative standard deviations (RSD) were calculated. For the analysis of MS data, the Waters QuanLynx software was used. Each described sample was prepared in triplicate and analyzed at least three times.

\section{Results}

\section{Preparation of LP standards}

Depending on the culture conditions, $\mathrm{PF}$ is produced by $P$. fluorescens $\mathrm{BD} 5$ as a mixture of up to four analogs: $\mathrm{PF} 1$ $\left(\mathrm{C}_{16}-\mathrm{Val}_{8}\right), \mathrm{PF} 2\left(\mathrm{C}_{16^{-}}-\mathrm{Leu}_{8}\right), \mathrm{PF} 3\left(\mathrm{C}_{18^{-}} \mathrm{Val}_{8}\right)$, and PF4 $\left(\mathrm{C}_{18^{-}}\right.$ $\mathrm{Leu}_{8}$ ). The analogs can be purified together, but the separation of each analog is also possible (Janek et al. 2010; Biniarz et al. 2015b). Similarly, SU produced by B. subtilis is a mixture of several analogs, which differ in the length of the acyl chain (from $\mathrm{C} 12$ to $\mathrm{C} 17$ ), as well as in amino acid substitutions (Sigma-Aldrich Surfactin-Product Information; Isa et al. 2008). The Sigma-Aldrich SU standard is sold as such a mixture (Sigma-Aldrich). The relative abundance of SU analogs was determined based on UPLC-MS TIC chromatograms (Fig. 1): (C12) 4.2\%, (C13) 11.8\%, (C14) 39.1\%, (C15) $35.5 \%$, (C16) $7.1 \%$, and (C17) $2.3 \%$. PF isolated from the culture A supernatant consisted primarily of PF2 (67.5\%) and PF3 (28.0\%), whereas PF1 (1.0\%) and PF4 (3.4\%) were observed in trace quantities (Fig. 1). The PF peaks from semipreparative HPLC were collected together, dried, weighed, and dissolved in $\mathrm{MeOH}$. These samples were then used as a $\mathrm{PF}$ standard for the designated experiments (Fig. 1). A mixture of $\mathrm{PF}$ analogs was used as a comparison to the $\mathrm{SU}$ mixture.

\section{Development of HPLC/UPLC-MS methods for LP quantification}

HPLC and UPLC-MS methods for two different HPLC systems and one UPLC-MS system were developed and optimized for the separation and quantification of LPs, using PF and SU as models. Gradient elution programs allowed us to analyze PF and SU standards with a resolution sufficient for separating individual LP isoforms. $\mathrm{Up}$ to $4 \mathrm{PF}$ isoforms and as many as $13 \mathrm{SU}$ analogs were identified by the UPLC-MS system (Fig. 1). Relatively short analysis times have been achieved with conventional HPLC systems. The analysis time was only $15 \mathrm{~min}$ for $\mathrm{PF}$ and $20 \mathrm{~min}$ for $\mathrm{SU}$, including the column wash and equilibration steps. Transferring these methods to UPLC-MS enabled 3.75-fold and 3.64-fold reductions of the analysis time for $\mathrm{PF}$ and $\mathrm{SU}$, respectively (Fig. 1). In addition, the system 3 MS TIC allowed not only the highly sensitive detection and quantification of LPs (Table 1) but also the characterization of individual LP isoforms based on molecular mass (Fig. 1). HPLC and UPLC-MS systems showed diverse sensitivity, linearity ranges, LOD, and LOQ for LPs (Table 1). All of the tested LC systems showed broad ranges $(7.81-500 \mathrm{mg} / \mathrm{ml}$ at the minimum) for $\mathrm{PF}$ and SU quantification when the absorbance at $210 \mathrm{~nm}$ was monitored. For MS TIC detection, the quantification range was narrow: between 3.91 and $125 \mathrm{mg} / \mathrm{L}$ for $\mathrm{PF}$ and 7.81 and $125 \mathrm{mg} / \mathrm{L}$ for SU. At the same time, the highest sensitivity $(0.98 \mathrm{mg} / \mathrm{L})$ was achieved with MS TIC detection (Table 1).

\section{Recovery of LP from standard solutions during DA}

The main aim of our work was to determine the recovery levels of LPs quantified with different LC Systems. This goal was achieved by adding known amounts of LP standards to LB medium and comparing LC measurements with standard curves. Three different concentrations of LPs $(50,100$, and $250 \mathrm{mg} / \mathrm{ml}$ ) were used to determine whether the concentration of LPs in a sample can affect the ability to obtain reliable and quantitative results. We also investigated the influence of solvent mixtures used to dissolve LP standards. LB medium and solutions of LB:water or LB:MeOH $(50: 50 v / v)$ were used to dissolve LPs.

The LC systems showed different LP recovery levels, ranging from $0 \%$ for system 3 to $100 \%$ for systems 1 and 2 (Table 2). We detected an increase in the recovery of LPs when LB:MeOH was used to dissolve the LP standards. For example, for $50 \mathrm{mg} / \mathrm{ml}$ PF quantified with system $3 \mathrm{DAD}$, the recovery of LP was only $8.0 \%$ in LB solution; in contrast, the recovery of LP was $62.1 \%$ when LB:MeOH was used as a solvent. A similar but weaker 

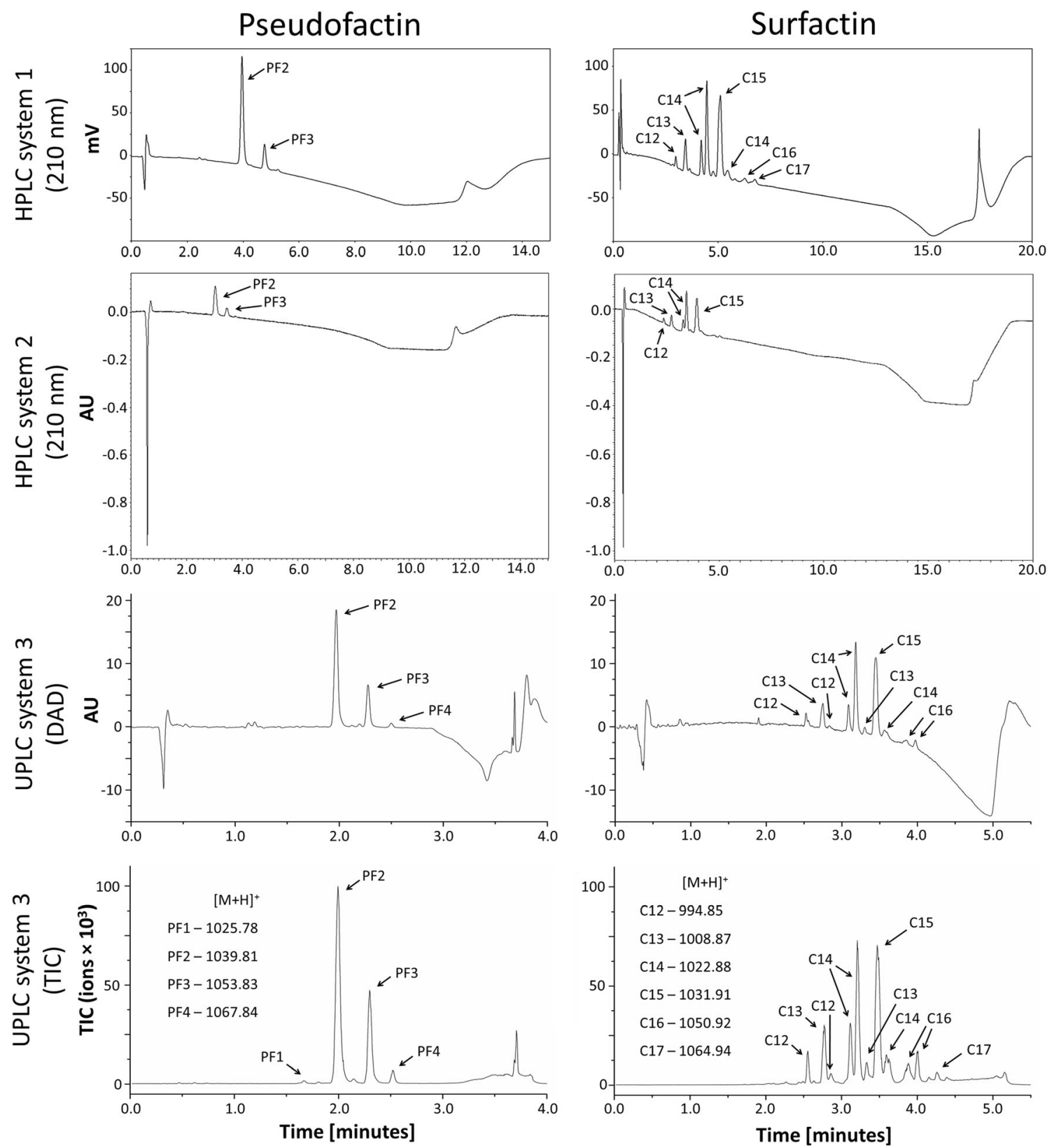

Fig. 1 Comparison of PF and SU separation with HPLC and UPLC-MS Systems. PF and $\mathrm{SU}$ standards were dissolved in $\mathrm{MeOH}$ prior to analysis. Essential peaks are marked with arrows and described. The observed $\mathrm{m} / \mathrm{z}$

effect was also observed for systems 1 and 2 (Table 2). Moreover, recovery was concentration-dependent, as higher recovery was observed for more concentrated LP solutions (Table 2). Systems 1 and 2 showed high and very high recovery of LPs (from 78.0 to $103.4 \%$ ) when LB:MeOH (50:50) was used as a solvent, whereas only $36.3-72.8 \%$ of the LP was detected with system 3 (Table 2). Recovery differences were also observed between PF and SU, particularly for system 3. PF in LB:MeOH, quantified with system 3, showed a $6.1-20.0 \%$ higher recovery than SU (Table 2). ratios of proton adducts for LP analogs $\left.\left([\mathrm{M}+\mathrm{H}]^{+}\right]\right)$are shown in the bottom panel

\section{Influence of $\mathrm{MeOH}$ concentration on $\mathrm{LP}$ recovery during DA}

UPLC-MS (system 3) showed the lowest recovery levels for LP (even when 50:50 LB:MeOH was used as a solvent) (Table 2). Therefore, we tested solvent mixtures of LB: $\mathrm{MeOH}$ with $\mathrm{MeOH}$ concentrations from 10 to $90 \%$ to investigate the influence of the $\mathrm{MeOH}$ concentration on the recovery levels of PF and SU quantified with system 3 . We showed that the recovery of LP increases with the $\mathrm{MeOH}$ concentration, reaching approximately $100 \%$ when $>80 \%$ 
Table 1 Calibration data for PF and SU quantified with various LC systems

\begin{tabular}{llllll}
\hline LP & LC system & Linearity range $(\mathrm{mg} / \mathrm{L})$ & $r^{2}$ & LOD $(\mathrm{mg} / \mathrm{L})$ & LOQ $(\mathrm{mg} / \mathrm{L})$ \\
\hline PF & System 1 & $3.91-1000$ & 0.9997 & 1.95 & 3.91 \\
& System 2 & $7.81-500$ & 0.9995 & 1.95 & 3.72 \\
& System 3 DAD & $7.81-500$ & 0.9981 & 3.91 & 7.81 \\
& System 3 MS TIC & $3.91-125$ & 0.9958 & 0.98 & 3.91 \\
SU & System 1 & $3.91-1000$ & 0.9998 & 1.95 & 3.91 \\
& System 2 & $7.81-500$ & 0.9997 & 1.95 & 7.81 \\
& System 3 DAD & $7.81-500$ & 0.9993 & 3.91 & 7.81 \\
& System 3 MS TIC & $7.81-125$ & 0.9951 & 0.98 & 7.81 \\
\hline
\end{tabular}

MeOH was used to dissolve a sample (Fig. 2). Interestingly, for solutions with low concentration of LPs $(50 \mathrm{mg} / \mathrm{ml})$ in up to $60 \% \mathrm{MeOH}$, the recovery of PF was 3.8-19.4\% higher than observed for SU. The opposite effect was observed for the higher concentrations analyzed $(250 \mathrm{mg} / \mathrm{ml})$, but only for solutions containing up to $20 \% \mathrm{MeOH}$. Here, SU recovery was 5.1-5.9\% higher than observed for PF (Fig. 2).

\section{DA of LPs in bacterial cultures}

PF and SU were produced by $P$. fluorescens BD5 and $B$. subtilis Natto KB1, respectively. Three oxygenation levels were used to culture the bacteria, resulting in various final LP concentrations in the cultures (Table 3). This approach allowed us to avoid introducing differences in the composition of the culture media between samples. Next, the samples were clarified, and LPs were quantified with the LC systems. To determine the influence of the $\mathrm{MeOH}$ concentration on LP quantification directly in the bacterial cultures, we quantified LPs in samples diluted two times with water $(2 \times$ water) and two or ten times with $\mathrm{MeOH}$ $(2 \times \mathrm{MeOH}$ and $10 \times \mathrm{MeOH})$. The results were compared to undissolved (undiluted with $\mathrm{MeOH}$ ) samples ( $1 \times$ sample), which were set as $100 \%$.

We detected an increase in LP recovery levels (in comparison to undissolved samples) when the samples were diluted two times or ten times with $\mathrm{MeOH}$. For system 1, a $2 \times$ dilution with $\mathrm{MeOH}$ resulted in a $2.1-10.7 \%$ higher recovery of LPs, while a $10 \times$ dilution with $\mathrm{MeOH}$ increased the recovery of LPs from 6.8 to $19.0 \%$ in comparison to undissolved samples (Table 3). The influence of $\mathrm{MeOH}$ addition was intense for system 3. We observed approximately 1.3 to 2.7 times higher concentrations of LPs for samples diluted $2 \times$ with $\mathrm{MeOH}$ in comparison to undissolved samples when using system 3 DAD. Even more dramatically, a 3.5- to 6.1-fold higher concentration of LPs (in comparison to undissolved samples) was observed when the samples were diluted $10 \times$ with $\mathrm{MeOH}$ prior to system $3 \mathrm{DAD}$ analysis (Table 3).

\section{Efficiency of FDSE for sample pretreatment during quantitative analysis of LPs}

We also tested the efficiency of freeze-drying followed by solvent extraction (FDSE) for the quantitative analysis of LPs in biological samples. FDSE was developed as a rapid LP extraction protocol that can be used prior to quantitative LC analysis of LPs. The sample pretreatment process can potentially minimize RP-LC column damage (e.g., by proteins) and as a result, extend the column lifespan.

Similar to DA, LP standards $(250 \mathrm{mg} / \mathrm{ml})$ from stock solutions were added to LB medium. Then, the samples were freeze-dried, and the resulting pellet was extracted three times with equal volumes of $\mathrm{MeOH}, \mathrm{EtOH}, \mathrm{ButOH}, \mathrm{ACN}$, or EtOAc. Next, the solvents were evaporated, the pellet was resuspended in $\mathrm{MeOH}$, and the samples were analyzed using system 3 DAD.

The FDSE efficiency was comparable for PF extracted with $\mathrm{MeOH}, \mathrm{EtOH}$, and ButOH, reaching 69.0-80.5\% after a onestep extraction and 97.0-99.7\% after a two-step extraction. The third extraction did not influence the final extraction efficiency (Fig. 3a). ACN and EtAC were less effective after onestep extraction, but approximately $90 \%$ of the PF was extracted after the double extraction process (Fig. 3a). The recovery levels of SU extracted by $\mathrm{MeOH}$ and $\mathrm{EtOH}$ were 75.9 and $84.1 \%$, respectively, after a one-step extraction. Nearly $100 \%$ of the SU was recovered after double extraction (Fig. 3b). EtAC was the less effective solvent for SU extraction-only $51.0,40.1$, and $7.6 \%$ of the SU was recovered after extraction (Fig. 3b). We also tested FDSE for low concentrations of LPs $(50 \mathrm{mg} / \mathrm{ml})$ and detected no significant influence of the LP concentration on the extraction efficiency by FDSE (data not shown). A similar protocol was applied for clarified bacterial cultures. Cultures of $P$. fluorescens BD5 and B. subtilis KB1 were analyzed. LPs in the cultures were initially quantified via system 3 (DAD) after diluting the samples $10 \times$ with $\mathrm{MeOH}$ (qf. Fig. 2 and Table 3). The measured concentrations were $490.0 \pm 8.5 \mathrm{mg} / \mathrm{ml}$ for PF and $1124.6 \pm 15.3 \mathrm{mg} / \mathrm{L}$ for SU. The FDSE efficiency values for PF and SU extracted from bacterial cultures were comparable to the FDSE efficiency values for samples containing LP standards (qf. Figs. 3 and 4). 
Approximately $100 \%$ of the LPs were recovered from samples double-extracted with $\mathrm{MeOH}$, EtOH, ButOH, and ACN. EtAC was less effective and extracted 94.5 and $89.1 \%$ of the $\mathrm{PF}$ and SU, respectively, after a two-step extraction.

\section{Discussion}

The complexity of biological matrices makes the quantification of active substances a challenging task (van den Broek et al. 2008). Over the years, BS have been quantified indirectly using various techniques, such as surface tension measurements or meniscus shape analysis, as examples. Due to the complexity of biological matrices, the results obtained with these indirect techniques are believed to be only semi-quantitative at best (Youssef et al. 2004; Burch et al. 2010; Marchant and Banat 2014; Rudden et al. 2015; Biniarz et al. 2016). Direct methods for the quantification of BS have been developed as an alternative. Perhaps the best known of these methods is the orcinol method, which has been proposed for the quantification of rhamnolipids. However, that method also suffers from crosstalk with medium components (e.g., glucose), which may lead to the overestimation of BS yields in culture medium (Marchant and Banat 2014; Rudden et al. 2015). Simple colorimetric methods were recently proposed for the detection and quantification of SU, but these methods have been cited in a limited number of research works to date (Zhu et al. 2014). Therefore, the reliable quantification of BS, particularly LPs, remains a challenging task.

LC is a powerful tool for the quantification and identification of various substances in biological samples. Recently, several reports have shown the possibility of using RPHPLC and RP-UPLC methods for the quantification and characterization of BS with high accuracy, sensitivity, and repeatability (Hsieh et al. 2008; Rao et al. 2013; Rudden et al. 2015). LC quantification of BS is typically preceded by sample pretreatment, primarily acid precipitation or solvent extraction. Next, the extracts are dried, dissolved in organic solvent (usually $\mathrm{MeOH}$ ), and analyzed via LC (Hsieh et al. 2008; Yokota et al. 2012; Marchant and Banat 2014; Zhu et al. 2014). These protocols are time-consuming and are therefore not applicable for high-throughput analysis. In addition, the recovery levels of BS usually remain unknown (Hsieh et al. 2008; Ke et al. 2015; Geissler et al. 2016).

Recently, LC and LC-MS methods for the quantification of rhamnolipids have been proposed and validated (Rudden et al. 2015). There are also a few examples of different protocols for sample pretreatment prior to LC quantification of LPs. In addition, method validation has been carried out in some cases. Yokota et al. quantified iturin A with HPLC and compared the recovery levels of this LP extracted from Bacillus culture supernatants using three methods: acid precipitation and $\mathrm{MeOH}$ extraction (APME), ButOH extraction and $\mathrm{MeOH}$ substitution 


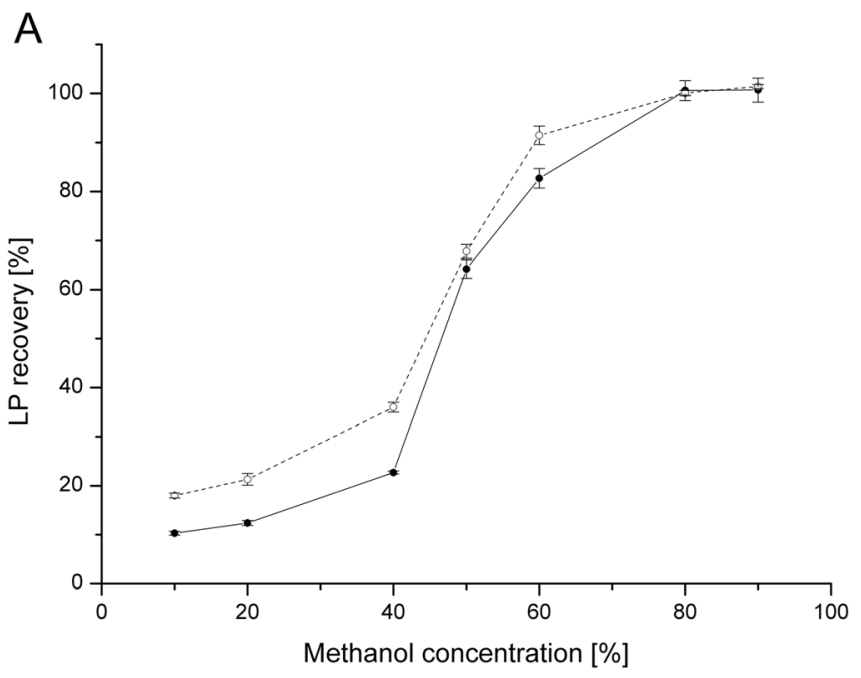

Fig. 2 Influence of $\mathrm{MeOH}$ concentration (percent) on the recovery levels of PF (a) and SU (b) quantified with system 3 (UPLC-MS). Two concentrations of LPs were used: $50 \mathrm{mg} / \mathrm{ml}$ (closed circles, solid lines)

(BEMS), and DA. Those authors showed poor recovery levels of iturin A for DA and APME ( 0.5 and $14.1 \%$, respectively) and high efficiency for BEMS (99.6\%) (Yokota et al. 2012). In contrast, Mubarak et al. showed that the recovery levels of SU quantified directly (DA) by HPLC were 94.1-102.4\%, but no information concerning the HPLC system used or the protocol used to prepare the samples for the recovery tests were included (Mubarak et al. 2015). Bie et al. used traditional acid precipitation to pellet raw antimicrobial substances produced by Bacillus sp. fmbJ and then tested four solvents $(\mathrm{MeOH}$, $\mathrm{EtOH}$, propanol, and $\mathrm{ButOH}$ ), as well as various $\mathrm{pH}$ levels and time periods, for the optimal extraction of active substance(s). Those authors found $\mathrm{MeOH}$ and $\mathrm{EtOH}$ to be efficient solvents. In addition, the $\mathrm{pH}$ and extraction time also affected the extraction efficiency (Bie et al. 2005). Liquid-liquid extraction (LLE) is widely used for the semi-preparative purification of LPs (Janek et al. 2010; Smyth et al. 2014), but only in a few research works LLE has been applied for analytical scale extraction. The reasons for this limitation are primarily poor recovery and the complexity of small-scale LLE (Smyth et al. 2010; Burch et al. 2010). An aqueous two-phase system (ATPS) was previously tested as an alternative to liquid-liquid extraction (LLE) to overcome the poor recovery of LLE (Yuan et al. 2011). Iturin A was quantified with good resolution, a relatively short analysis time, and high accuracy and recovery (up to $97 \%$ ), but the simplicity and feasibility of using the proposed method in high-throughput quantification is, in our opinion, arguable (Yuan et al. 2011). Solid-phase extraction (SPE) has been also implemented for the quantification of LPs in bacterial cultures. Here, $\mathrm{C}_{8}$ or $\mathrm{C}_{18}$ SPE columns are typically used. Clarified culture supernatants are applied to the SPE column, and LPs are then eluted with $\mathrm{MeOH}$. Then, the methanolic extracts are analyzed via LC (Gancel et al. 2009; Coutte et al. 2010).

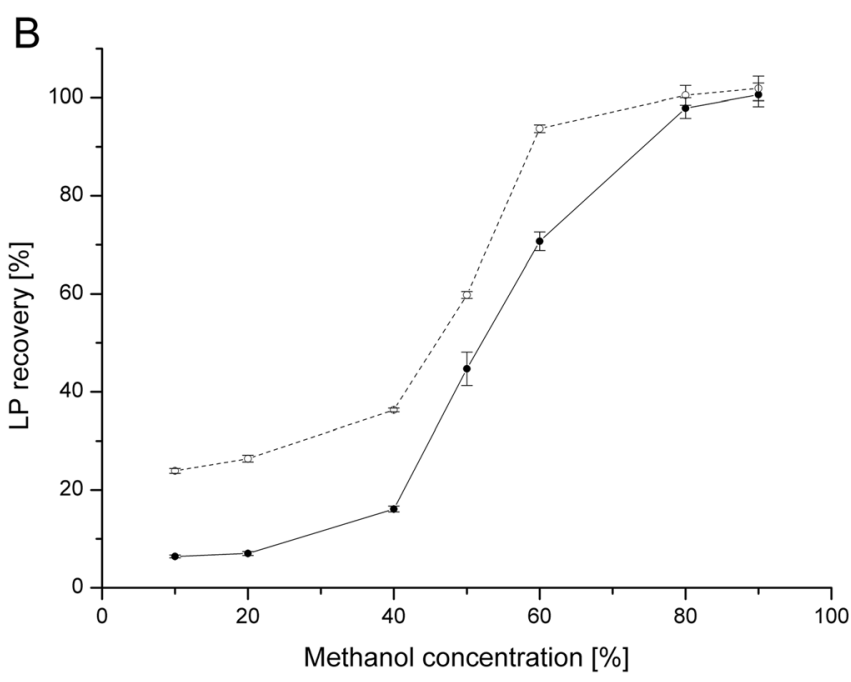

and $250 \mathrm{mg} / \mathrm{ml}$ (open circles, dashed lines). LPs were quantified with DAD. Quantification with MS TIC showed similar patterns (data not shown)

In our work, we developed and validated a simple method for sample pretreatment (FDSE). Several dozen samples can be lyophilized and extracted in only a few hours. In addition, except the lyophilizer, no specialized equipment or consumables (such as an SPE chamber or SPE columns) are needed. At the same time, FDSE provides high recovery levels for LPs (more than 97\% for samples twofold extracted with $\mathrm{MeOH}, \mathrm{EtOH}, \mathrm{ButOH}$, and $\mathrm{ACN}$ ) and good repeatability. Therefore, this method can be used for the extraction of LPs from cultivation medium prior to LC or LC-MS identification and LP quantification.

Sample pretreatment complicates and increases the cost of LP quantification and therefore should be minimized in highthroughput optimization of LP production or LP analysis in the food industry or healthcare products. The direct injection of microbial cultures onto LC columns for the quantification of LPs appears to be the solution to this problem, but this approach has been mentioned only in a few research works (Lin et al. 2007; Isa et al. 2007; Rao et al. 2008; Yokota et al. 2012; Yi et al. 2016). Thus, DA of LPs should be validated, especially because recovery problems have been reported previously (Yokota et al. 2012). Simple dilution of the sample with an organic solvent (e.g., $\mathrm{MeOH}$ or $\mathrm{EtOH}$ ) could be a solution. Sample dilution with $\mathrm{MeOH}$ followed by DA via HPLC was tested for iturin A quantification (Rao et al. 2008). Culture samples were clarified by centrifugation and filtration. Then, the cell-free supernatant was passed through 1000 and $10 \mathrm{kDa}$ ultrafiltration membranes, and the concentrated fraction was diluted ten times with methanol and analyzed via HPLC (Rao et al. 2008). In another report, those authors also diluted the samples ten times with a different solvent system (acetonitrile: $10 \mathrm{mM}$ ammonium acetate, 40:60 v/v) and omitted the ultrafiltration steps (Lin et al. 2007). HPLC methods showed good resolution for iturin A isomers. In addition, SU was directly quantified via HPLC after diluting the 


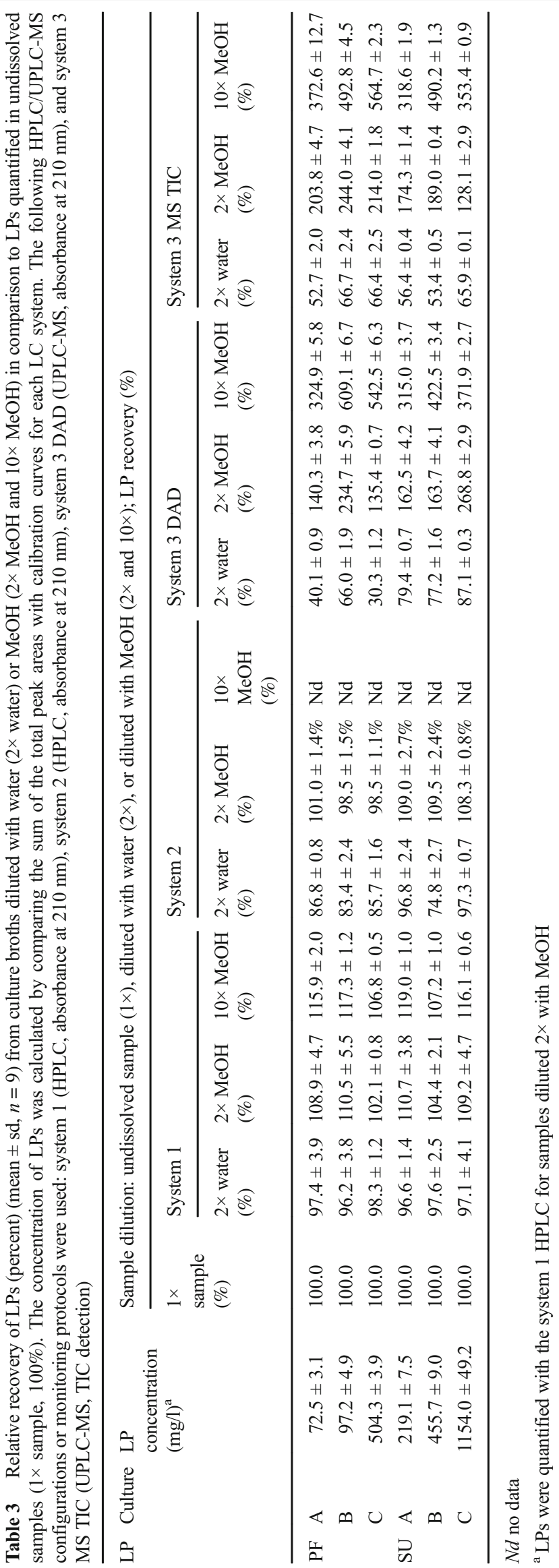

samples with $\mathrm{MeOH}$ (Yi et al. 2016). Unfortunately, no recovery tests or method validation were performed by the authors (Lin et al. 2007; Rao et al. 2008; Yi et al. 2016).

We observed that the accuracy of direct LP quantification in biological samples via LC depends on the composition of the sample injected, especially the LP concentration and the solvent mixture used to dissolve a sample $(\mathrm{MeOH}$ concentration in a sample). This effect can be probably explained by the loss of LPs caused by their adsorption on surfaces of the LC-MS system, as well as consumables used for sample pretreatment (e.g., pipette tips or Eppendorf tubes) (van den Broek et al. 2008). Similar effects were previously observed for bioactive peptides in various complex matrices (e.g., in human serum). The adsorption of peptides on surfaces (e.g., glass and plastic vials, pipette tips, and inner parts of LC-MS systems) is a well-known phenomenon. It was also confirmed that several factors can influence the adsorption of peptides on surfaces, primarily solvent properties ( $\mathrm{pH}$, ionic strength, etc.), the concentration and physiochemical properties of the peptide, temperature, and the nature of interphase (e.g., container material) (van den Broek et al. 2008). The same effects can probably also be observed for LPs, considering the similar chemical structures and physiochemical properties of various bioactive peptides and LPs. This phenomenon should be investigated in the future.

As pointed out by Rudden et al., there is a great need for the development of fast, accurate, and reliable analytical methods for the quantification of BS. In addition, such methods should be standardized between laboratories to make the BS yields reported in research works more comparable (Rudden et al. 2015). Previously, UPLC-MS/MS was developed and properly validated for the quantification of rhamnolipids (Rudden et al. 2015). To our knowledge, no LC method has been properly developed and validated for the quantification and characterization of LPs. Thus, the main objective our work was to develop such a method. Recently, high-performance and highaccuracy thin layer chromatography (HPTLC) was evaluated and validated for the simultaneous quantification of SU, iturin A, and fengycin directly from B. subtilis cultures (Geissler et al. 2016); however, in our opinion, the LC device is more ubiquitous in analytical laboratories than HPTLC. Therefore, the development of LC methods for the quantification of LPs is of great importance.

Sample analysis with HPLC can be time-consuming. This limitation also concerns the quantification of LPs. In several research papers, HPLC analysis of lipopeptides varied from 20 to up to $100 \mathrm{~min}$ per sample (Lin et al. 2007; Isa et al. 2007; De Bruijn et al. 2008; Yokota et al. 2012; Willenbacher et al. 2014; Zhu et al. 2014). In contrast, up to 20 samples containing SU, iturin A, and fengycin can be quantified simultaneously with HPTLC in 80 min (Geissler et al. 2016). Our HPLC methods allow us to quantify LPs in a relatively short time (15 min for PF and 20 min for SU). Transferring these methods to UPLC-MS reduced the analysis time to $4 \mathrm{~min}$ for 


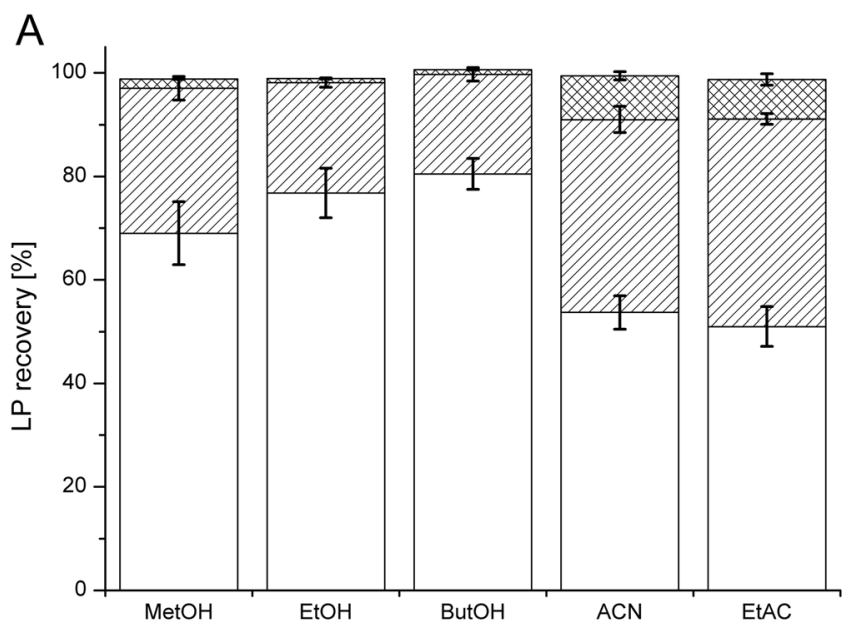

Fig. 3 Recovery of PF (a) and SU (b) standards ( $250 \mathrm{mg} / \mathrm{ml})$ from LB medium after FDSE. The extraction stages are shown as different patterns: first extraction (white bars), second extraction (hatched bars), and third extraction (crossed bars). Five solvents were used to extract LPs

$\mathrm{PF}$ and $5.5 \mathrm{~min}$ for SU. These times make our UPLC-MS methods for the quantification of LPs high-throughput, allowing the analysis of a large number of samples in relatively short time, with high accuracy and precision. Simultaneously, the use of the LC-MS system allows not only the separation of individual LPs' structural analogs but also the precise identification of these compounds.

In our work, we also identified an issue with poor recovery of LPs quantified directly in culture samples. This observation can probably be explained by the adsorption of LPs on surfaces (e.g., plastic consumables or LC system parts). Moreover, we proposed and evaluated a simple solution for this issue, which is the modification of a sample with $\mathrm{MeOH}$ prior to LC analysis. Our results indicate that the $\mathrm{MeOH}$ concentration in the sample should reach $80 \%$ or more to

A

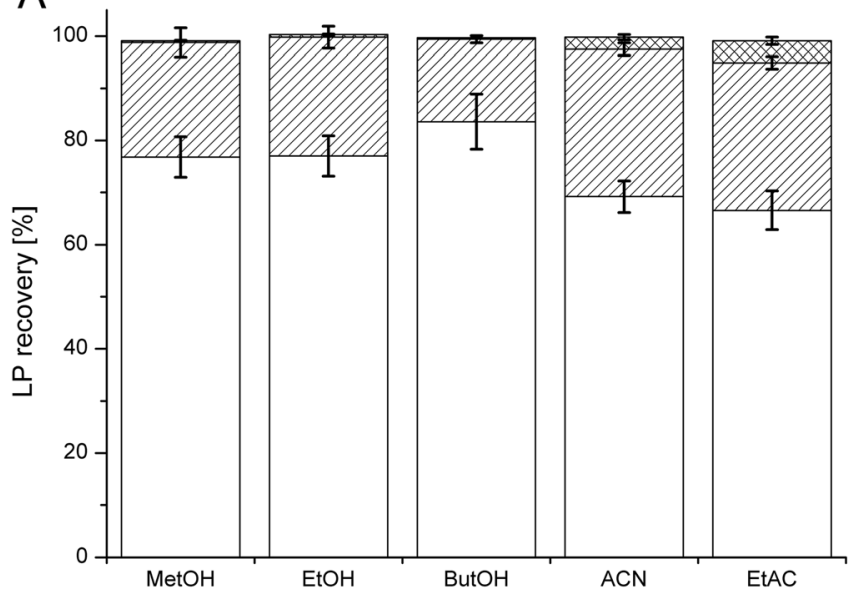

Fig. 4 Recovery of PF (a) and SU (b) from bacterial cultures $(490.0 \pm 8.5 \mathrm{mg} / \mathrm{L}$ PF and $1124.6 \pm 15.3 \mathrm{mg} / \mathrm{L} \mathrm{SU})$ after FDSE. The extraction stages are shown as different patterns: first extraction (white bars), second extraction (hatched bars), and third extraction (crossed
B

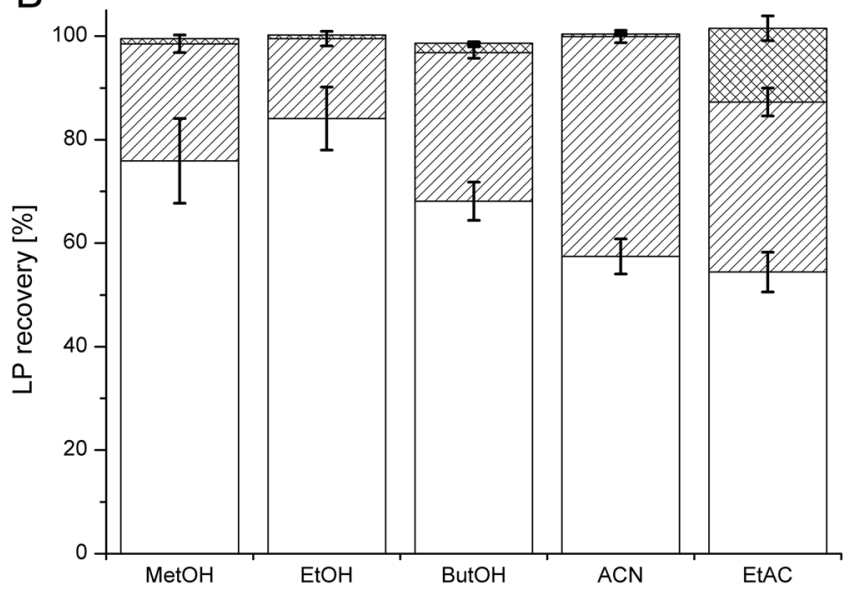

from lyophilized samples: methanol $(\mathrm{MeOH})$, ethanol (EtOH), butanol $(\mathrm{ButOH})$, acetonitrile $(\mathrm{ACN})$, and ethyl acetate (EtAC). LPs were quantified with system $3 \mathrm{DAD}$

completely avoid adsorption issues. Therefore, we suggest researchers working on LPs to validate their LC quantification methods, especially to investigate recovery of LPs (as shown in our work). Application of our simple method with adding $\mathrm{MeOH}$ to clarified culture supernatants prior quantification should be also checked for other classes of LPs. We hope that our work will be a starting point for the development of standardized and properly validated high-throughput LC methods for the quantification of LPs.

In summary, we showed:

- Novel, accurate HPLC and UPLC-MS methods for the direct quantification of lipopeptides in culture supernatants have been developed and validated, using pseudofactin and surfactin as model molecules.

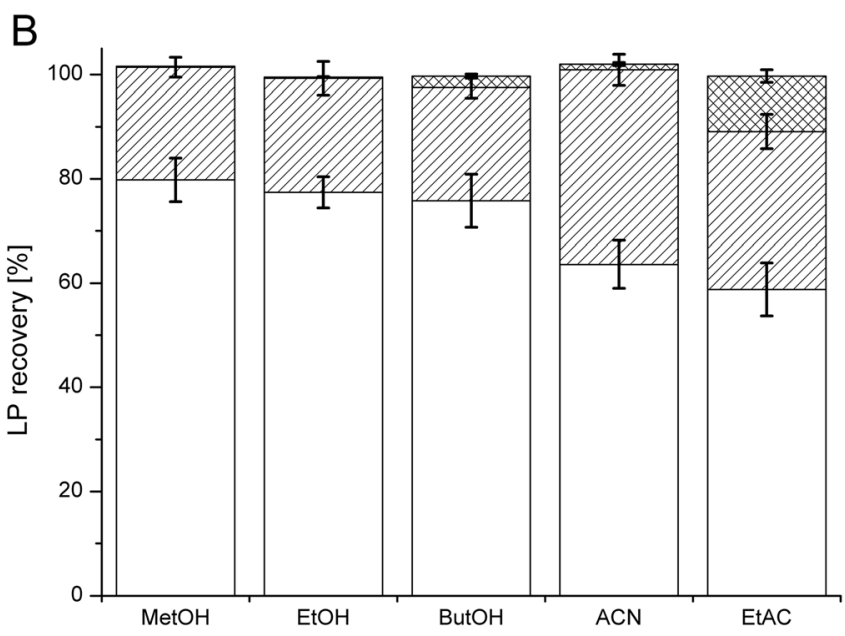

bars). Five solvents were used to extract LPs from lyophilized culture samples: methanol $(\mathrm{MeOH})$, ethanol $(\mathrm{EtOH})$, butanol (ButOH), acetonitrile $(\mathrm{ACN})$, and ethyl acetate (EtAC). LPs were quantified with system 3 
- Pseudofactin and surfactin can be quantified via HPLC in 15 and 20 min per sample, while UPLC-MS reduced these times to 4 and $5.5 \mathrm{~min}$ per sample, respectively.

- A high accuracy of direct lipopeptide quantification via LC can be achieved by diluting the samples with methanol.

- Lipopeptides'structural analogs can be separated and identified using a MS detector.

- Culture supernatants can be freeze-dried, and lipopeptides can be extracted from the resulting pellet with organic solvents (methanol, ethanol, butanol, or acetonitrile).

Acknowledgments We thank the Department of Genetic Biochemistry, Faculty of Biotechnology, University of Wroclaw, Poland, for allowing us to use the Waters Acquity UPLC-MS. We also thank the Department of Inorganic Chemistry, Faculty of Pharmacy with Subfaculty of Laboratory Medicine, Medical University of Gdansk, Poland, for allowing us to use the Waters HPLC system (e2695 pump, 2998 PDA).

\section{Compliance with ethical standards}

Conflict of interest The authors declare that they have no conflict of interest.

Funding This work was supported by the National Science Centre, Poland grant 2016/20/T/NZ1/00536 and by The Leading National Research Center (KNOW) program of the Wroclaw Center of Biotechnology for the years 2014-2018.

Ethical approval This article does not contain any studies with human participants performed by any of the authors.

Open Access This article is distributed under the terms of the Creative Commons Attribution 4.0 International License (http:// creativecommons.org/licenses/by/4.0/), which permits unrestricted use, distribution, and reproduction in any medium, provided you give appropriate credit to the original author(s) and the source, provide a link to the Creative Commons license, and indicate if changes were made.

\section{References}

Akpa E, Jacques P, Wathelet B, Paquot M, Fuchs R, Budzikiewicz H, Thonart P, Conditions C (2001) Influence of culture conditions on lipopeptide production by Bacillus subtilis. Appl Biochem Biotechnol 91-93:551-561. doi:10.1385/ABAB:91-93:1-9:551

Banat IM, Franzetti A, Gandolfi I, Bestetti G, Martinotti MG, Fracchia L, Smyth TJ, Marchant R (2010) Microbial biosurfactants production, applications and future potential. Appl Microbiol Biotechnol 87: 427-444. doi:10.1007/s00253-010-2589-0

Ben Ayed H, Hmidet N, Béchet M, Chollet M, Chataigné G, Leclère V, Jacques P, Nasri M (2014) Identification and biochemical characteristics of lipopeptides from Bacillus mojavensis A21. Process Biochem 49:1699-1707. doi:10.1016/j.procbio.2014.07.001

Bie X, Lu Z, Lu F, Zeng X (2005) Screening the main factors affecting extraction of the antimicrobial substance from Bacillus sp. fmbJ using the Plackett-Burman method. World J Microbiol Biotechnol 21:925-928. doi:10.1007/s11274-004-6722-Z

Biniarz P, Baranowska G, Feder-Kubis J, Krasowska A (2015a) The lipopeptides pseudofactin II and surfactin effectively decrease
Candida albicans adhesion and hydrophobicity. Antonie Van Leeuwenhoek. doi:10.1007/s10482-015-0486-3

Biniarz P, Krasowska A, Łukaszewicz M (2015b) Ratio of isomers of pseudofactin, a lipopeptide produced by Pseudomonas fluorescens BD5, changes in response to different carbon and nitrogen sources. J Biotechnol 208:S116. doi:10.1016/j.jbiotec.2015.06.366

Biniarz P, Łukaszewicz M, Janek T (2016) Screening concepts, characterization and structural analysis of microbial-derived bioactive lipopeptides: a review. Crit Rev Biotechnol 8551:1-18. doi:10. 3109/07388551.2016.1163324

van den Broek I, Sparidans RW, Schellens JHM, Beijnen JH (2008) Quantitative bioanalysis of peptides by liquid chromatography coupled to (tandem) mass spectrometry. J Chromatogr B Anal Technol Biomed Life Sci 872:1-22. doi:10.1016/j.jchromb.2008.07.021

Burch AY, Shimada BK, Browne PJ, Lindow SE (2010) Novel highthroughput detection method to assess bacterial surfactant production. Appl Environ Microbiol 76:5363-5372. doi:10.1128/AEM. 00592-10

Chen CY, Baker SC, Darton RC (2007) The application of a high throughput analysis method for the screening of potential biosurfactants from natural sources. J Microbiol Methods 70:503510. doi:10.1016/j.mimet.2007.06.006

Coutte F, Lecouturier D, Yahia SA, Leclère V, Béchet M, Jacques P, Dhulster P (2010) Production of surfactin and fengycin by Bacillus subtilis in a bubbleless membrane bioreactor. Appl Microbiol Biotechnol 87:499-507. doi:10.1007/s00253-010-2504-8

Das P, Mukherjee S, Sivapathasekaran C, Sen R (2010) Microbial surfactants of marine origin: potentials and prospects. Adv Exp Med Biol 672:88-101

Davis DA, Lynch HC, Varley J (2001) The application of foaming for the recovery of surfactin from B. subtilis ATCC 21332 cultures. Enzym Microb Technol 28:346-354. doi:10.1016/S0141-0229(00)00327-6

De Bruijn I, de Kock MJD, De Waard P, van Beek T a, Raaijmakers JM (2008) Massetolide a biosynthesis in Pseudomonas fluorescens. J Bacteriol 190:2777-2789. doi:10.1128/JB.01563-07

De Faria AF, Teodoro-Martinez DS, De Oliveira Barbosa GN, Gontijo Vaz B, Serrano Silva Í, Garcia JS, Tótola MR, Eberlin MN, Grossman M, Alves OL, Regina Durrant L (2011) Production and structural characterization of surfactin $(\mathrm{C} 14 / \mathrm{Leu} 7)$ produced by Bacillus subtilis isolate LSFM-05 grown on raw glycerol from the biodiesel industry. Process Biochem 46:1951-1957. doi:10.1016/j. procbio.2011.07.001

Duarte C, Gudiña EJ, Lima CF, Rodrigues LR (2014) Effects of biosurfactants on the viability and proliferation of human breast cancer cells. AMB Express 4:40. doi:10.1186/s13568-014-0040-0

Gancel F, Montastruc L, Liu T, Zhao L, Nikov I (2009) Lipopeptide overproduction by cell immobilization on iron-enriched light polymer particles. Process Biochem 44:975-978. doi:10.1016/j.procbio. 2009.04.023

Geissler M, Oellig C, Moss K, Schwack W, Henkel M, Hausmann R (2016) High-performance thin-layer chromatography (HPTLC) for the simultaneous quantification of the cyclic lipopeptides Surfactin, Iturin a and Fengycin in culture samples of Bacillus species. J Chromatogr B. doi:10.1016/j.jchromb.2016.11.013

Gudiña EJ, Rangarajan V, Sen R, Rodrigues LR (2013) Potential therapeutic applications of biosurfactants. Trends Pharmacol Sci 34:667675. doi:10.1016/j.tips.2013.10.002

Guez JS, Müller CH, Danze PM, Büchs J, Jacques P (2008) Respiration activity monitoring system (RAMOS), an efficient tool to study the influence of the oxygen transfer rate on the synthesis of lipopeptide by Bacillus subtilis ATCC6633. J Biotechnol 134:121-126. doi:10. 1016/j.jbiotec.2008.01.003

Hsieh F-CC, Lin T-CC, Meng M, Kao S-SS (2008) Comparing methods for identifying Bacillus strains capable of producing the antifungal lipopeptide iturin a. Curr Microbiol 56:1-5. doi:10.1007/s00284007-9003-x 
Isa M, Coraglia D, Frazier R, Jauregi P (2007) Recovery and purification of surfactin from fermentation broth by a two-step ultrafiltration process. J Memb Sci 296:51-57. doi:10.1016/j.memsci.2007.03.023

Isa MHM, Frazier R a, Jauregi P (2008) A further study of the recovery and purification of surfactin from fermentation broth by membrane filtration. Sep Purif Technol 64:176-182. doi:10.1016/j.seppur. 2008.09.008

Jajor P, Piłakowska-Pietras D, Krasowska A, Łukaszewicz M (2015) Surfactin analogues produced by Bacillus subtilis strains grown on rapeseed cake. J Mol Struct:1-6. doi:10.1016/j.molstruc.2016.02.014

Janek T, Łukaszewicz M, Rezanka T, Krasowska A (2010) Isolation and characterization of two new lipopeptide biosurfactants produced by Pseudomonas fluorescens BD5 isolated from water from the Arctic archipelago of Svalbard. Bioresour Technol 101:6118-6123. doi:10. 1016/j.biortech.2010.02.109

Janek T, Łukaszewicz M, Krasowska A (2012) Antiadhesive activity of the biosurfactant pseudofactin II secreted by the Arctic bacterium Pseudomonas fluorescens BD5. BMC Microbiol 12:24. doi:10. 1186/1471-2180-12-24

Janek T, Krasowska A, Radwańska A, Łukaszewicz M (2013) Lipopeptide biosurfactant pseudofactin II induced apoptosis of melanoma a 375 cells by cpecific interaction with the plasma membrane. PLoS One 8:1-9. doi:10.1371/journal.pone.0057991

Janek T, Rodrigues LR, Gudiña EJ, Czyżnikowska Ż (2016) Structure and mode of action of cyclic lipopeptide pseudofactin II with divalent metal ions. Colloids Surfaces B Biointerfaces 146:498-506. doi:10.1016/j.colsurfb.2016.06.055

Joshi S, Suthar H, Yadav A, Hingurao K, Nerurkar A (2013) Occurrence of biosurfactant producing Bacillus spp. in diverse habitats

Jurado E, Altmajer D, Gudi EJ, Teixeira J a, Rodrigues LR, Vaz DA, Gudiña EJ, Alameda EJ, Teixeira J a, Rodrigues LR (2012) Performance of a biosurfactant produced by a Bacillus subtilis strain isolated from crude oil samples as compared to commercial chemical surfactants. Colloids Surfaces B Biointerfaces 89:167-174. doi: 10.1016/j.colsurfb.2011.09.009

Ke WJ, Hsueh YH, Cheng YC, Wu CC, Liu ST (2015) Water surface tension modulates the swarming mechanics of Bacillus subtilis. Front Microbiol 6:1-12. doi:10.3389/fmicb.2015.01017

Kowall M, Vater J, Kluge B, Stein T, Franke P, Ziessow D (1998) Separation and characterization of surfactin isoforms produced by Bacillus subtilis OKB 105. J Colloid Interface Sci 204:1-8. doi:10. 1006/jcis. 1998.5558

Lin H, Koteswara Y, Wu W, Tzeng Y (2007) Ferrous ion enhanced lipopeptide antibiotic iturin a production from Bacillus amyloliquefaciens B128. Int J Appl Sci Eng 5:123-132

Marchant R, Banat IM (2014) Protocols for measuring biosurfactant production in microbial cultures. In: Hydrocarbon and Lipid Microbiology Protocols

Mnif I, Ghribi D (2015) Review lipopeptides biosurfactants: mean classes and new insights for industrial, biomedical, and environmental applications. Biopolymers 104:129-147. doi:10.1002/bip.22630

Mubarak MQE, Hassan AR, Hamid AA, Khalil S, Isa MHM (2015) A simple and effective isocratic HPLC method for fast identification and quantification of surfactin. Sains Malaysiana 44:115-120

Mukherjee AK, Das K (2010) Microbial surfactants and their potential applications: an overview. Adv Exp Med Biol 672:54-64

Mukherjee S, Das P, Sen R (2009) Rapid quantification of a microbial surfactant by a simple turbidometric method. J Microbiol Methods 76:38-42. doi:10.1016/j.mimet.2008.09.010

Mulligan CN (2005) Environmental applications for biosurfactants. Environ Pollut (Barking, Essex 1987) 133:183-198. doi:10.1016/j. envpol.2004.06.009

Raaijmakers JM, de Bruijn I, de Kock MJ (2006) Cyclic lipopeptide production by plant-associated Pseudomonas spp.: diversity, activity, biosynthesis, and regulation. Mol Plant-Microbe Interact 19:699 710
Rao KY, Lin H, Wu W, Tzeng Y (2008) Evaluation of HPLC and MEKC methods for the analysis of lipopeptide antibiotic iturin a produced by Bacillus amyloliquefaciens. Int J Appl Sci Eng 6:85-96

Rao M, Wei W, Ge M, Chen D, Sheng X (2013) A new antibacterial lipopeptide found by UPLC-MS from an actinomycete Streptomyces sp. HCCB10043. Nat Prod Res 27:2190-2195. doi: 10.1080/14786419.2013.811661

Romero D, de Vicente A, Rakotoaly RH, Dufour SE, Veening J-W, Arrebola E, Cazorla FM, Kuipers OP, Paquot M, Pérez-García A (2007) The iturin and fengycin families of lipopeptides are key factors in antagonism of Bacillus subtilis toward Podosphaera fusca. Mol Plant-Microbe Interact 20:430-440. doi:10.1094/MPMI-20-4-0430

Rudden M, Tsauosi K, Marchant R, Banat IM, Smyth TJ (2015) Development and validation of an ultra-performance liquid chromatography tandem mass spectrometry (UPLC-MS/MS) method for the quantitative determination of rhamnolipid congeners. Appl Microbiol Biotechnol 99:9177-9187. doi:10.1007/s00253-015-6837-1

Satpute SK, Bhawsar BD, Dhakephalkar PK, Chopade BA (2008) Assessment of different screening methods for selecting biosurfactant producing marine bacteria. Indian JHournal Mar Sci 37:243-250

Satpute SK, Banpurkar AG, Dhakephalkar PK, Banat IM, Chopade BA (2010) Methods for investigating biosurfactants and bioemulsifiers: a review. Crit Rev Biotechnol 30:127-144. doi:10.3109/ 07388550903427280

Smyth TJ, Rudden M, Tsaousi K, Marchant R, Banat IM (2014) Protocols for the isolation and analysis of lipopeptides and bioemulsifiers. In: Springer Protocols Handbooks

Smyth TJP, Perfumo A, McClean S, Marchal R, Banat IM (2010) Isolation and analysis of lipopeptides and high molecular weight biosurfactants. In: Handbook of Hydrocarbon and Lipid Microbiology, pp 3687-3704

Soberon-Chavez G, Miller-Maier RM (2011) Biosurfactants: A general overview. In: Biosurfactants. From Genes to Applications, pp 1-11

Tang J-S, Zhao F, Gao H, Dai Y, Yao Z-H, Hong K, Li J, Ye W-C, Yao X-S (2010) Characterization and online detection of surfactin isomers based on HPLC-MSn analyses and their inhibitory effects on the overproduction of nitric oxide and the release of TNF- $\alpha$ and IL- 6 in LPS-induced macrophages. Mar Drugs 8:2605-2618. doi:10.3390/md8102605

US FDA (1999) Guidance for Industry, Validation of Analytical Procedures. Methodology

Willenbacher J, Zwick M, Mohr T, Schmid F, Syldatk C, Hausmann R (2014) Evaluation of different Bacillus strains in respect of their ability to produce surfactin in a model fermentation process with integrated foam fractionation. Appl Microbiol Biotechnol 98:9623-9632

Yi G, Liu Q, Lin J, Wang W, Huang H, Li S (2016) Repeated batch fermentation for surfactin production with immobilized Bacillus subtilis BS-37: two-stage $\mathrm{pH}$ control and foam fractionation. J Chem Technol Biotechnol. doi:10.1002/jctb.5028

Yokota K, Yatsuda M, Miwa E, Higuchi K (2012) Comperative study on sample preparation methods for the HPLC quantification of iturin from culture supernatant of an antagonistic Basillus strain. J Int Soc Southeast Asian Agric Sci 18:70-75

Youssef NH, Duncan KE, Nagle DP, Savage KN, Knapp RM, McInerney MJ (2004) Comparison of methods to detect biosurfactant production by diverse microorganisms. J Microbiol Methods 56:339-347. doi:10.1016/j.mimet.2003.11.001

Yuan J, Raza W, Huang Q, Shen Q (2011) Quantification of the antifungal lipopeptide iturin a by high performance liquid chromatography coupled with aqueous two-phase extraction. J Chromatogr B Analyt Technol Biomed Life Sci 879:2746-2750. doi:10.1016/j. jchromb.2011.07.041

Zhu L, Xu Q, Jiang L, Huang H, Li S (2014) Polydiacetylene-based highthroughput screen for surfactin producing strains of Bacillus subtilis. PLoS One 9:e88207. doi:10.1371/journal.pone.0088207 